\title{
Convergence of Highly Resolved and Rapid Screening Platforms with Dynamically Engineered, Cell Phenotype-Prescriptive Biomaterials
}

\author{
Neal K. Bennett ${ }^{1}$ - Anandika Dhaliwal ${ }^{1}$ - Prabhas V. Moghe ${ }^{1,2}$
}

Published online: 18 March 2016

(C) Springer International Publishing AG 2016

\begin{abstract}
Biophysical and biochemical cues from the cellular microenvironment initiate intracellular signaling through cellular membrane receptors and trigger specific cell developmental programs. Extracellular substrates and matrix scaffolds engineered to mimic cell's native physiological environment must incorporate the multifactorial parameters (composition, micro and nanoscale organization, and topography) of the extracellular matrix as well as the dynamic nature of the matrix. The design of such engineered biomaterials is challenged by the inherent complexity and dynamic nature of the cellextracellular matrix reciprocity, while the validation of robust microenvironments requires a deeper, higher content phenotypic resolution of cell-matrix interactions alongside a rapid screening capability. To this end, high-throughput platforms are integral to facilitating the screening and optimization of complex engineered microenvironments for directing desired cell developmental pathway. This review highlights the recent advances in biomaterial platforms that present dynamic cues and enable high throughput screening of cell's response to a combination of micro-environmental factors. We also address some newer techniques involving high-content image informatics to elucidate emergent cellular behaviors with a focus on stem cell regenerative endpoints.
\end{abstract}

This article is part of the Topical Collection on Drug Delivery

Neal K. Bennett and Anandika Dhaliwal contributed equally to this work.

Prabhas V. Moghe

moghe@rutgers.edu

1 Department of Biomedical Engineering, Rutgers University, 599 Taylor Road, Piscataway, NJ 088534, USA

2 Department of Chemical and Biochemical Engineering, Rutgers University, Piscataway, NJ, USA
Keywords High throughput screening $\cdot$ High-content imaging $\cdot$ Extracellular matrix $\cdot$ Dynamic scaffolds

\section{Introduction}

The primary goal of regenerative medicine is to recreate human tissues, either to increase understanding of human physiology or pathophysiology, to screen for effective therapeutics, or to replace lost tissue. Key to tissue regeneration or replacement is the effective combination of relevant lineage-specific cells and a supportive biomaterial. Biomaterials used in tissue engineering approaches play a major role of influencing a wide range of cell behavior, including cell viability, differentiation, motility, and apoptosis. These changes in cell behavior are often sensitively modulated by changes in the biomaterial's properties like topography, stiffness, and material chemistry. Most studies to date have examined cellular response to alterations in specific individual biomaterial parameters $[1,2]$. Given the multi-factorial nature of physiologic extracellular matrices, several recent reports indicate that the most favorable cell-niche incorporates a combination of biologically active, microenvironmental cues [3-5]. Further, prevalent studies tend to focus on studying the influence of static topographical, chemical, or mechanical cues on cell behavior. However, dynamic matrix changes are important to a number of biological processes including tissue formation, regeneration, and pathophysiological processes such as tumor growth [6-8]. Therefore, there exists a need to develop biomaterial platforms that can address the limitations in previously developed biomaterials by firstly allowing evaluation of dynamic cues, and secondly by facilitating screening of a combination of cues.

Recent advances in stimuli-responsive materials [9-11], as well as the increased recognition of the need to combine programmable cues with dynamic cell behaviors, have led to the 
development of a number of highly innovative biomaterial configurations. Additionally, technological developments such as advances in nanoscale and microsystem technologies have also enabled the fabrication of novel high-throughput platforms to screen varied topographies and geometries [12-14], or to identify an optimal combination of bioactive cues $[15,16]$. These high-throughput biomaterial platforms specifically seek to accelerate the development of materials for cell-based applications by designing materials that elicit desired cell behavior, through rapid and parallel screening of cell behaviors in response to a wide range of potentially relevant microenvironmental cues and substrate characteristics. Such platforms have many salient features that include reproducibility, suitability to automation, usage of lesser cells and reagents for screening, and cost effectiveness.

Here, we review a newer generation of biomaterials, which leverage the influence of topography, stiffness, and chemistry on cell behavior in innovative ways, particularly focusing on dynamic and high-throughput platforms, as well as highcontent analysis approaches to study cells on these platforms (Fig. 1).

\section{Dynamic Platforms to Model-Changing Cell Environments}

Functional tissue engineering requires a precise control over the spatio-temporal single-cell and multicellular organization and lineage-specific behavior through biomaterial-induced cues. This precise control requires the use of materials that combine desirable mechanical, topographical, and chemical cues, and allow for manipulation of these attributes to mimic the dynamic cell environment. Much of this work has been accomplished through developments with stimuli responsive materials, which change form in response to an external stimuli, such as mechanical strain, light, or change in temperature or $\mathrm{pH}[17,18]$. Previous studies have used these materials to investigate the effects of changing topography on cell shape, which is mediated by integrin receptor redistribution and cytoskeletal remodeling and ultimately governs cell functions including proliferation, differentiation, or death [19, 20]. However, only in recent studies have efforts been made to decouple effects of dynamic strain and deformation on cellular shape. For example, Pholpabu et al. [21] used a pre-strained elastomeric substrate, $\mathrm{SiO}_{2}$-coated polydimethylsiloxane (PDMS), to produce a substrate capable of alternating between three distinct topographies [21]. The alternation between flat, parallel wavy, or perpendicular wavy grooves was induced in less than $3 \mathrm{~s}$, and by mechanical strain values lower than $3.5 \%$, which is the threshold strain detected to date by mammalian cells.. The authors observed rapid and robust changes in fibroblast morphology when cultured on substrates switched from flat to wavy features, but no significant changes to gross steady-state morphology on substrates switched from flat to wavy to flat. These results suggest that the presentation of low-strain induced, reversible topographical cues allowed the authors to decouple changes in cell morphology based on contact guidance with those induced by substrate deformation.

While oxidized PDMS and shape-memory polymers have allowed for the isolation and investigation of key cellbiomaterial interactions, these materials are restricted to a limited range of topographies [17, 18]. An alternative approach was taken by Kiang et al. [22], who developed a soft polyacrylamide hydrogel with randomly embedded magnetic nickel microwires, allowing reversible changes in surface roughness from $\Delta R_{\mathrm{RMS}}$ of 0.05 to $0.70 \mu \mathrm{m}$ in response to variations in magnetic field [22]. Using this system, the authors found that vascular smooth muscle cells displayed morphological changes in response to acute but not cyclic changes in topography. Another substrate with tunable topography was developed by Kirschner and Anseth [23] who used poly(ethylene glycol) (PEG) hydrogels with photolabile linkages, allowing for the creation of topographical features through precise spatial erosion [23]. They demonstrated that the creation of channels in this substrate led to a significant increase in cellular aspect ratio of human mesenchymal stem cells, which were reversed with the creation of perpendicular channels within the same substrate. These findings could be used to direct localized stem cell function and fate in vitro for transplantation in cell therapies, and guide the creation of dynamic implantable devices.

Mechanical stimuli are an important variety of cues encountered by cells within the body, which can impact cell motility, proliferation, and differentiation [24, 25]. A wide spectrum of biological processes is characterized by changes in stiffness of tissue, including in tissue development [26], stress from locomotion and weight bearing in the musculoskeletal system, or arterial stiffening in atherosclerosis [27]. Several approaches have studied the role of spatiotemporal control over mechanical properties on cultured cell environment mediated, for example, through the formation or cleavage of crosslinking sites within flexible polymer-based gels $[28,29]$. Parallel studies that focus on the use of photocleavable o-nitrobenzyl group to reduce material stiffness [30, 31] have been reviewed elsewhere [11]. Other strategies to control material stiffness in situ have included the use of a secondary crosslinked network or interpenetrating network (IPN), where the two gel networks independently contribute a tunable, biologically inert, mechanical support component, and a biochemical cellular support component [32, 33]. Tong and Yang [33] demonstrated the utility of such a system as a stem cell niche by modulating osteogenic differentiation of human adipose-derived stem cells on an IPN consisting of a biochemical support network presenting varied RGD concentrations and a mechanical support network with mechanical stiffness ranging from 0.9 to $91.3 \mathrm{kPa}$ [33]. 
Fig. 1 Schematic of the convergence of three promising approaches in advancing understanding of cell-biomaterial interaction for tissue engineering applications: dynamic cell scaffolds that possess cell-matrix reciprocity, high throughput technologies for comprehensive rapid screening, and high-content cellular analysis based on phenotypic image informatics

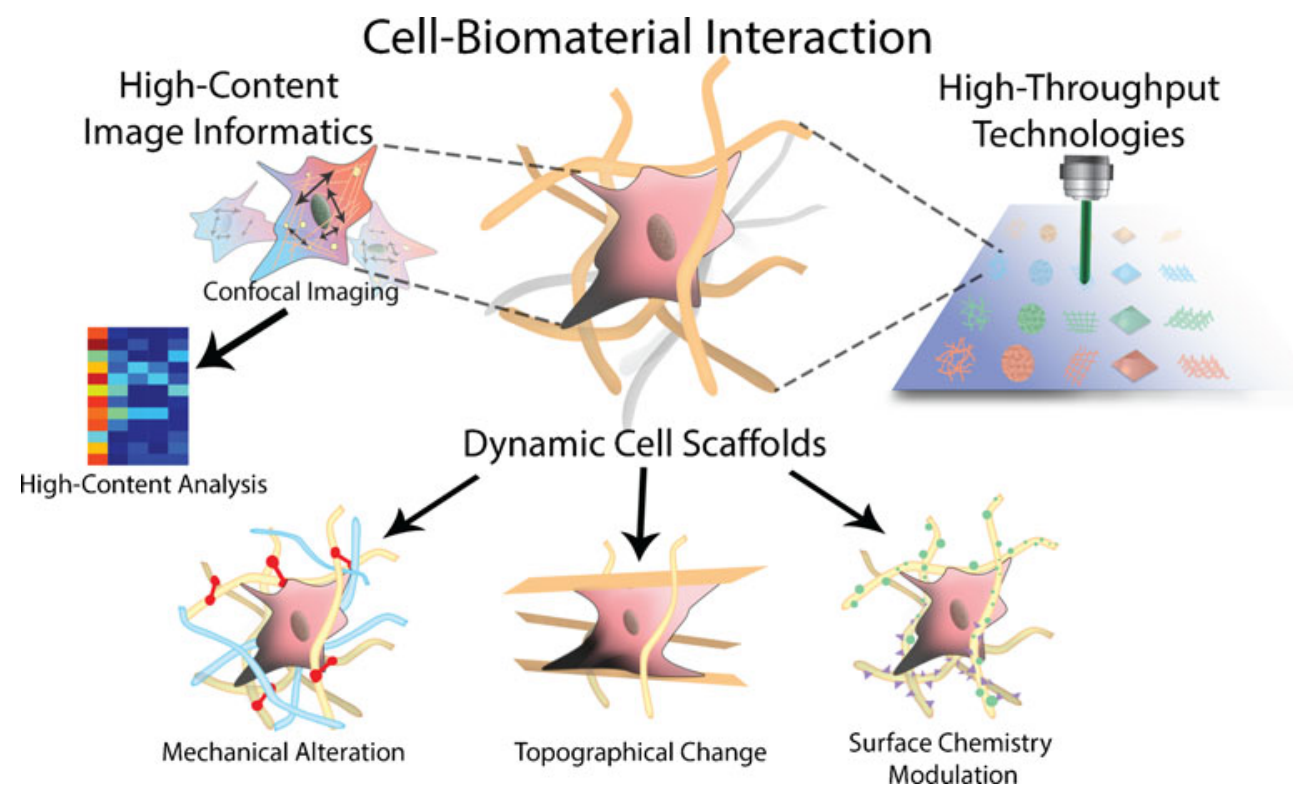

Mechanical network cross-linking could be conducted in physiological conditions, and matrix metalloproteinasesensitive peptides could be incorporated, allowing for dynamic mechanical stiffness properties in situ. They furthermore demonstrated the versatility of the IPN platform by producing the same mechanical stiffness using polyacrylamide, linear PEG-diacrylate, and multi-arm PEG for the mechanical support network in conjunction with a constant biochemical support network.

While a majority of tissue scaffolds generally display linear elastic properties, materials with strain-thickening or shearthinning mechanical behavior allow for material self-healing and injectability, as well as enhanced cell remodeling or communication through the exertion of traction forces. Winer et al. characterized a strain-thickening fibrin gel which extended the range of intercellular communication and allowed for the formation of multicellular patterns on the order of $100 \mu \mathrm{m}$, which was not observed using linear elastic materials [34]. Fibroblasts or human mesenchymal stem cells cultured on these fibrin gels generated local stiffness by exerting strain, resulting in dramatically increased cell spreading and the ability to mechanically interact with other cells at distances over four times their cell diameter. Many self-assembled materials rely on multiple noncovalent intermolecular interactions, and the temporary disruption and re-establishment of these interactions confers shear-thinning dynamic mechanical behavior. A number of self-assembling peptide-based hydrogels [35, 36], peptide hybrid materials employing a combination of peptides and chemical functionalities with known assembly properties [37], and protein or large polypeptide materials [38] with shear-thinning behavior have been observed to support cell growth and guide cell development, which has been reviewed elsewhere [39]. A novel non-peptide material was developed by Gaffey et al., who created a hyaluronic acidbased self assembling and shear thinning hydrogel, stabilized by non-covalent host-guest complexes between adamantine and $\beta$-cyclodextrin [40]. This substrate was demonstrated to support the culture of endothelial progenitor cells and enhance the survival of their transplantation into rat myocardium. In this case, the shear-thinning material not only supported the culture and delivery of encapsulated cells, but its mechanically dynamic nature also enabled it to conform to targeted host tissue and enhance host tissue regeneration, even in the absence of transplanted cells. This material was observed to increase vasculogenesis and improve ventricular function, showing promise for myocardial infarct treatment. Together, these studies represent key advances in understanding how materials with dynamic mechanical properties interact with both cultured cells and also host tissue.

Many studies have used technology available for over a decade to create scaffolds with dynamic adhesion properties through triggered changes in surface chemistry [33, 41], which have been the subject of multiple reviews [11, 42]. A substantial number of materials trigger cell behaviors such as migration or differentiation through controlled release of soluble factors, usually through tunable degradation properties or simple diffusion [43, 44], which have also been reviewed extensively $[45,46]$. However, a number of biological processes including adult differentiation, embryonic tissue formation, and immunological response to infection antigens depend on tight temporal regulation of signaling molecules [47-50]. As one example, transplantable poly-lactide-coglycolide matrices with controlled release of granulocytemacrophage colony-stimulating factor (GM-CSF) were designed to promote initial recruitment of dendritic cells to the implanted scaffold and initiate differentiation over the course 
of 2 weeks [51]. More recent advances in reversible presentation of biomolecules help in moving the field closer to mimicking these key biological processes, as opposed to relying on the typically irreversible approaches. One approach used three orthogonal chemistries to reversibly pattern PEG hydrogels with proteins of interest [52]: first, PEG hydrogels were generated with pendant alkoxyamines masked by photocleavable 2-(2-nitrophenyl)propyloxycarbonyl (NPPOC) groups; next, proteins pre-treated with a photocleavable o-nitrobenzyl group tethered to an aldehyde are introduced into the hydrogels after NPPOC groups are released, liberating alkoxyamines to react with the protein-linked aldehyde groups; finally, o-nitrobenzyl groups linking the protein of interest to the hydrogel can be cleaved. This platform was used to culture human mesenchymal stem cells into osteoblasts in a temporally and regionally defined manner. This approach represents a useful tool to further engineer changes in cell behavior through a dynamically controlled biochemical environment.

A number of key challenges must be overcome to develop biomaterials with a robust dynamic interaction with living cells. The introduction of external stimuli designed to induce topographical, chemical, or mechanical change in biomaterials must be within the range of tolerable conditions for cultured cells and may influence the baseline cell behavior [53]. As discussed earlier, recent studies have focused on minimizing the effects of external stimuli, by using stimuli that are either below cellular thresholds for detection, or that cells are incapable of detecting. Future studies may increase focus on biomaterials that autonomously transition between known states, which would eliminate the influence of these external stimuli. While current dynamic biomaterials are far from recapitulating the complex and changing in vivo cellular environment, recent innovations have generated tools to aid in fundamental studies of time-dependent cell-biomaterial interactions, and will guide the design of biomaterials engineered for precise cellular responses and tissue formation.

\section{High-Throughput Platforms to Screen Complex Cell-Niches}

High-throughput platforms are designed to screen cell behaviors in response to a wide range of potentially relevant conditions in order to elucidate a specific subset of biomaterial features that elicit desired cell behavior. One of the earliest examples of high throughput platforms is the application of polymeric material arrays for high-throughput surface characterization (HTSC) [54-56]. Over the last decade, HTSC has been applied to elucidate the relationship between polymeric structure or surface chemistry and biological function including cell adhesion and differentiation [57-59], to identify the ideal bioactive polymer design in large material libraries and accelerate the discovery of new biomedical materials. Highthroughput surface analysis, including time-of-flight secondary ion mass spectrometry (ToF-SIMS), X-ray photoelectron spectroscopy (XPS), and water contact angle (WCA) measurements have been effectively employed to determine surface characteristics and correlations between surface chemistry and cell activity $[56,58,60]$. Typically, high-content analysis of fluorescently labeled biological markers either absorbed directly on the array or stained in cells attached to polymer spots is also employed in high-throughput platforms to assess cell-material interactions. HTSC approaches and microarray production have been reviewed previously [54-56]. As exciting avenues for accelerated biomaterial discovery, these platforms also generate data that can be used to elucidate correlations between surface chemical structure and cellular behavior that could not have been predicted by a rational design approach based simply on review of the bulk or surface structure [55].

Advances in nano-scale and micro-scale technologies have accelerated the fabrication of high-throughput materials incorporating varied topographies and surface features [12-14]. For example, a 'Biochip' was fabricated containing 41 nanopatterns and employed it to systematically evaluate and identify nanotopographical cues like depth, width, (an)isotropy and spacing (ridge-groove ratio) that tightly regulate osteoblast behavior [61]. Specifically, this report demonstrated that the rate of cell motility is determined by ridge width, with the osteoblasts being most motile on a ridge-to-groove ratio (R:G) of 1:3 and a pitch of $400 \mathrm{~nm}$ [61]. On the other hand, to identify topographies inducing specific morphologies, MSC proliferation and osteogenesis, large libraries of parameterized topographical material surfaces, referred to as "TopoChips" and comprising over 2100 distinct topographies, have been fabricated and systematically applied [14, 62]. Through these studies, features controlling cell behavior were determined that can aid in designing surfaces that promote osteointegration around implants, or osteogenesis. However, all these studies have been largely restricted to assessing optimal material properties by modulating a single material characteristic such as material chemistry or topography.

Recent studies have evidenced that a convergence of various physical, chemical, and mechanical cues are required to elicit desired cell response [3-5]. The interplay between substrate stiffness and geometry in guiding osteogenic differentiation was demonstrated in the Killian laboratory by employing microcontact printing of adhesion proteins on polyacrylamide gels of variable stiffness [4]. When cells were confined to shapes promoting cytoskeletal tension, increased osteogenesis was observed across substrates with increasing stiffness [4]. For studies focused on investigating biochemical cues, the importance of considering the mechanical properties of a target tissue in concert with biochemical niche signals in vitro was illustrated by Gobaa et al. [3]. Using a 
microarray-based platform, they showed that the most favorable effect of a specific protein on adipogenesis manifests at a specific range of stiffness [3]. The sustained development of biomaterials therefore calls for screening strategies that combine the application of diverse biomaterial cues along with dynamically modulated high-throughput evaluation.

Many recent advances have also been made in the development of such high-throughput platforms that comprise variations of a combination of characteristics, and have been summarized in Table 1. Nakajima et al. [63] fabricated a biomaterial platform representing immobilized extracellular matrix (ECM) proteins as well as growth factors using photoassisted patterning of an alkanethiol self-assembled monolayer, and employed it to detect microenvironment cues for neural stem cell (NSC) proliferation and differentiation [63]. Laminin-1, fibronectin, ProNectin, and poly(ethyleneimine) were identified to maximally promote NSC adhesion, while cell proliferation was found to be maximally promoted on spots containing immobilized EGF [63]. In another notable study, Gobaa et al. [15] reported a microengineered platform comprised of soft hydrogel microwell arrays encompassing hydrogels with modular stiffness (shear moduli of 1-50 kPa) with combinations of proteins spotted by robotic technology [15]. They applied this platform to identify artificial niches influencing adipogenic and osteogenic differentiation of human mesenchymal stem cells (MSCs) [3, 15], as well as supporting self-renewal of non-adherent mouse neural stem cells (NSCs) [15, 16]. Garcia-Cordero et al. [67] fabricated a microfluidic platform that quantified cytokine production from dendritic cells stimulated with ten different adjuvants alone and in pairwise combinations, at multiple concentrations, and identified synergistic adjuvant effects that were recapitulated in vivo [67]. These platforms are instrumental in discerning the underlying synergistic effects of various biomaterial properties.
Another class of emerging high-throughput screening studies are based on culturing and evaluating cells in 3D microenvironments, mimicking the physiological dimensionality [64, 65]. Floren and Tan [64] developed a high-throughput ECM platform based on polyethylene (PEGDM) electrospun fibrous scaffolds incorporating 64 unique combinations of 6 ECM proteins, namely collagen I, collagen III, collagen IV, laminin, fibronectin, and elastin, in-conjunction with substrate stiffness of 4.6 or $20 \mathrm{KPa}$ [64]. Using this platform, specific neotissue microenvironments were identified to instruct MSC adhesion, spreading and vascular lineage commitment. In a parallel investigation, 3D combinatorial niches were fabricated by Memic and Khademhosseini [65] by utilizing a robotic microarray spotter to rapidly print droplets consisting of MSCs, gelatin methacrylate, prepolymer solution, and various ECM proteins on TMSPMA functionalized glass slide, and applied to identify optimal niche for osteogenesis [65]. Such platforms are especially useful in designing scaffolds for 3D and in-vivo applications.

\section{High-Content Image Informatics for Timely Screening and Cell State Profiling}

Conventional analyses to assess the outcome of cellbiomaterial interactions were accomplished by tracking protein expression using immunocytochemistry (for example SDS PAGE, ELISA) or using mass spectrometry (for example, LC-MS/MS). These methods are largely cell populationoutputs, and are time consuming and laborious, especially when performed to profile cell response to multiple conditions in a high-throughput platform simultaneously or at multiple time-points in dynamic micro-environments. Another limitation of these methods is that they are endpoint in nature and fail to detect and analyze cell response to external cues within the

Table 1 Recent advances in high throughput biomaterial platforms evaluated a combination of parameters influencing biological processes

\begin{tabular}{|c|c|c|c|}
\hline Fabrication method & Parameters tested & Biological process evaluated & Reference \\
\hline Electron beam lithography (EBL) & 40 nano-patterns/topographies & Osteoblast behavior & {$[61]$} \\
\hline Photolithography, hot embossing & TopoChip with 2176 topographies & $\begin{array}{l}\text { Cell morphology, MSC proliferation } \\
\text { and osteogenesis }\end{array}$ & {$[14,62]$} \\
\hline $\begin{array}{l}\text { Photo-assisted patterning of an alkanethiol self-assembled } \\
\text { monolayer }\end{array}$ & $\begin{array}{l}\text { ECM proteins and growth factor } \\
\text { combinations }\end{array}$ & $\begin{array}{l}\text { NSC adhesion, proliferation and } \\
\text { differentiation }\end{array}$ & {$[63]$} \\
\hline $\begin{array}{l}\text { Electrospun fibrous scaffolds with ECM protein } \\
\text { microarrays }\end{array}$ & Six ECM proteins, two substrate stiffness & $\begin{array}{l}\text { MSC adhesion, spreading and } \\
\text { differentiation }\end{array}$ & {$[64]$} \\
\hline $\begin{array}{l}\text { Hydrogels with protein microarrays spotted } \\
\text { using robotic printing }\end{array}$ & $\begin{array}{l}\text { Shear moduli of } 1-50 \mathrm{kPa} \text {, protein } \\
\text { combinations }\end{array}$ & $\begin{array}{l}\text { Adipogenic and osteogenic differentiation } \\
\text { of MSCs, NSC differentiation and } \\
\text { renewal }\end{array}$ & {$[3,15,16]$} \\
\hline $\begin{array}{l}\text { Robotic microarray spotter to spot precursor } \\
\text { gel solution }\end{array}$ & ECM proteins & $\begin{array}{l}\text { MSC proliferation and osteogenic } \\
\text { differentiation }\end{array}$ & {$[65]$} \\
\hline Light-modulated hyaluronic acid hydrogels & Ligand density, substrate stiffness & Cell behavior & {$[66]$} \\
\hline Photolithography & $\begin{array}{l}10 \text { adjuvants alone and pairwise at } 3 \\
\text { concentrations, } 435 \text { conditions }\end{array}$ & Inflammatory cytokine production & {$[67]$} \\
\hline
\end{tabular}


first $0-72 \mathrm{~h}$ after exposure. Recently incorporation of high content image analysis approaches to evaluate cell response to engineered biomaterial platforms is gaining momentum. The Moghe lab at Rutgers has advanced the application of high-content image informatics (HCII) to deduce biomarker profiles at the single cell level indicative of emergent phenotypes on a variety of platforms [68-72]. This approach is based on acquiring high-resolution images of cellular reporters and performing high-content analysis to acquire features quantifying reporter organization, in tandem with machine learning approaches to model sub-cellular reporter organization. In a seminal report in 2010, we demonstrated that while cell shape alone could not distinguish emergent phenotypes within first $24 \mathrm{~h}$ of differentiation induction, higher-order variations in cell shape and cytoskeletal organization that occur within hours of stimulation can be employed to forecast the lineage commitment fates of human mesenchymal stem cells (hMSCs) seeded on fibronectin (Fn) [71]. In this study, a set of quantifiable morphometric features were formulated to define high-order variations in actin organization and employed with machine learning approaches to forecast cell phenotypes. Additionally, using these morphometric features the degree of osteogenic differentiation of hMSCs was predictably profiled on nine polymers with diverse physicochemical properties [71]. Employing high-content image informatics for cells with fluoro-reporters also allows for live-cell imaging and tracking cell-state changes in dynamic scaffolds. This approach has been illustrated by Treiser et al. [70] who have illustrated the methodology to apply HCII using green fluorescent protein (GFP) fusion genes to profile change in cell cytoskeleton and discern combinatorial variations in substrate composition [70].
Using a similar approach based on employing morphometric descriptors of green fluorescent protein reporter for farnesylation (GFP-f) in engineered Saos-2 cells, cell spreading and attachment was characterized in biomaterial substrates possessing gradients in surface topography [69].

Recent advances include the development of more sensitive surrogate markers based on nuclear reporters, for prospectively profiling phenotypic commitment. Specifically, the organizational metrics of interchromatin speckle factor SC-35 were used to profile hMSC lineage commitment and adhesion signaling in response to soluble cues and on varied platforms including patterned surfaces, fibrous scaffolds, and micropillars [72]. In this approach, texture features capturing minute high-order variations in the sub-nuclear spatial organization of SC-35 were computed and utilized with machine learning approaches to generate a predictive cell-state classification model. Using this methodology, differences in osteogenesis induced by varied topographies were quantitatively identified and classified for hMSCs cultured on a subset of micropillars chosen from a library of 2176 distinct, randomly designed surface topographies $[14,72]$. These studies, therefore, illustrate that high-content image informatics-based approaches provide complementary insights as they allow screening relevant materials in high-throughput and dynamic platforms by evaluating cell response at single cell level and at early time-points.

Many high-throughput platforms, high-content imaging, and analysis systems are now commercially available and have been listed in Table 2. For example, PolAn's microarray plates provide platforms functionalized with peptides or polymers, while Softwell plates from Matrigen provide a platform
Table 2 Commercially available high throughput platforms, high content imaging systems and analysis software
High-throughput commercially available platforms

References

1. PolyAn's microarry plates/slides, functionalised for DNA-, peptide- or protein microarrays.

2. Softwells from Matrigen, in multiwell format with nine levels of stiffness $(\mathrm{KPa})$

High-content/throughput imaging systems

1. PerkinElmer Operetta ${ }^{\circledR}$ High Content Imaging System

2. In Cell HCA systems (GE healthcare)

$[76,77]$

3. Cellomics ArrayScan VTI HCS platform (Thermo Scientific)

$[78,79]$

4. ImageXpress Micro XLS Widefield High-Content Analysis System (Molecular Devices)

Image analysis software supporting high-content analysis (HCA)

1. Harmony Software
2. Investigator
3. ImageJ
4. Cell Profiler
5. MetaMorph Software (Molecular
Devices)
6. Imaris
7. Image Pro Plus/Premier
Additional data analysis software
8. Spotfire TIBCO)

This software is used with the Operetta system. This software is used with In Cell HCA systems.

Open source software.

Open source software.

Can be used independently or along with imaging platforms.

$[86,87]$

Data visualization and analytics software. 
constituting substrates of varied stiffness for high-throughput screening and imaging. The high-throughput platforms require commercially available imaging systems that support highcontent imaging and analysis. The Cellomics ArrayScan platform is a fluorescent imaging platform with a live cell tracking module for high content imaging. The PerkinElmer Operetta High Content Imaging system and the In Cell HCA by GE Healthcare, are two commercially available confocal imaging systems for high-throughput image-based screening and analysis. These systems are automation compatible and can be employed for high-throughput studies to assess various cellular processes including cell migration, cell differentiation, cell morphology changes, and cytotoxicity in response to external cues. Harmony software is available to be used with the Operetta system, while the In Cell Investigator software is available for the In Cell HCA systems to conduct highcontent image analysis. Other software that can be employed for high-content image analysis include commercially available software Imaris, MetaMorph microscope automation and image analysis software, and Image Pro Plus or Premier, and open source software ImageJ and Cell Profiler.

Novel systems involving multiplexed methods are also being developed to facilitate high-throughput assays and screening applications. One such emerging technology is mass cytometry or CyTOF, which employs stable rare earth metal probes, enables multiplex of 40 independent measurements on single cells and can be employed for deep profiling of cells and high-throughput cell annotation based on phenotypic or functional characterization [88, 89]. Other emerging systems include Single molecule array $\left(\mathrm{Simoa}^{\mathrm{TM}}\right)$, Optimiser ${ }^{\mathrm{TM}}$, SQIDLite ${ }^{\mathrm{TM}}$, and $\mathrm{iLite}^{\mathrm{TM}}$. These technologies as well as CyTOF have been evaluated and comprehensively reviewed elsewhere [90].

\section{Conclusion}

In conclusion, the design and development of innovative biomaterials are continually inspired by the unraveling complexity of multifactorial and dynamic nature of extracellular matrix signaling behaviors. A number of new chemistry approaches as well as rapid screening methods are offering valuable insights for material scientists and biologists to develop optimized scaffold design principles, instruct optimal cell state development, and assess integrated "systems" parameters modulating key biological processes. These new technologies and next-generation dynamic biomaterials have demonstrated promising outcomes in a broad range of regenerative medicine and immunological applications, including enhanced cell transplantation, cancer targeting, and vaccine development. Recent advances in analytical methodologies employed image informatics in conjunction with high-content analysis and are proving to be a vital technology for robust cell profiling and biomaterial screening. We expect that the high-content analyses could be combined with the high-throughput screening platforms that (a) identify the most influential biomaterials configurations, and (b) better understand the dynamic and synergistic profiles of cell behaviors at a single cell level. Consequently, these dynamic platforms can most robustly recapitulate "heterogeneous biology-on-a-chip" and provide unprecedented insights into the local, molecular-level interplay between physical and chemical cues, the cellular reprogramming events, and the emergence of dominant phenotypic lineages of cells.

Acknowledgments This study was supported by funding from NIH EB001046 (RESBIO, Integrated Resources for Polymeric Biomaterials) and NSF DGE 0801620 (IGERT on Integrated Science and Engineering of Stem Cells).

\section{Compliance with Ethical Standards}

Conflict of Interest On behalf of all authors, the corresponding author states that there is no conflict of interest.

Human and Animal Rights and Informed Consent This article does not contain any studies with human or animal subjects performed by any of the authors.

\section{References}

1. Huang C, Dai J, Zhang XA. Environmental physical cues determine the lineage specification of mesenchymal stem cells. Biochim Biophys Acta. 2015;1850(6):1261-6. doi:10.1016/j.bbagen.2015. 02.011 .

2. Kwon HJ, Yasuda K. Chondrogenesis on sulfonate-coated hydrogels is regulated by their mechanical properties. J Mech Behav Biomed Mater. 2013;17:337-46. doi:10.1016/j.jmbbm. 2012.10.006.

3. Gobaa S, Hoehnel S, Lutolf MP. Substrate elasticity modulates the responsiveness of mesenchymal stem cells to commitment cues. Integr Biol. 2015;7(10):1135-42. doi:10.1039/c4ib00176a.

4. Lee J, Abdeen AA, Huang TH, Kilian KA. Controlling cell geometry on substrates of variable stiffness can tune the degree of osteogenesis in human mesenchymal stem cells. J Mech Behav Biomed Mater. 2014;38:209-18. doi:10.1016/j.jmbbm.2014.01.009.

5. Lee J, Abdeen AA, Zhang D, Kilian KA. Directing stem cell fate on hydrogel substrates by controlling cell geometry, matrix mechanics and adhesion ligand composition. Biomaterials. 2013;34(33): 8140-8. doi:10.1016/j.biomaterials.2013.07.074.

6. Bonnans C, Chou J, Werb Z. Remodelling the extracellular matrix in development and disease. Nat Rev Mol Cell Biol. 2014;15(12): 786-801. doi:10.1038/nrm3904nrm3904.

7. Naba A, Clauser KR, Lamar JM, Carr SA, Hynes RO. Extracellular matrix signatures of human mammary carcinoma identify novel metastasis promoters. Elife. 2014;3, e01308. doi:10.7554/eLife. 01308.

8. Pickup MW, Mouw JK, Weaver VM. The extracellular matrix modulates the hallmarks of cancer. EMBO Rep. 2014;15(12):1243-53. doi:10.15252/embr.201439246. 
9. Cheng CM, Steward Jr RL, LeDuc PR. Probing cell structure by controlling the mechanical environment with cell-substrate interactions. J Biomech. 2009;42(2):187-92. doi:10.1016/j.jbiomech. 2008.10.014S0021-9290(08)00520-4.

10. Guvendiren M, Burdick JA. Engineering synthetic hydrogel microenvironments to instruct stem cells. Curr Opin Biotechnol. 2013;24(5):841-6. doi:10.1016/j.copbio.2013.03.009S0958 1669(13)00066-9.

11. Kim J, Hayward RC. Mimicking dynamic in vivo environments with stimuli-responsive materials for cell culture. Trends Biotechnol. 2012;30(8):426-39. doi:10.1016/j.tibtech.2012.04. 003S0167-7799(12)00054-6.

12. Bae H, Chu H, Edalat F, Cha JM, Sant S, Kashyap A, et al. Development of functional biomaterials with micro- and nanoscale technologies for tissue engineering and drug delivery applications. J Tissue Eng Regen Med. 2014;8(1):1-14. doi:10.1002/term.1494.

13. Kim P, Epstein AK, Khan M, Zarzar LD, Lipomi DJ, Whitesides GM, et al. Structural transformation by electrodeposition on patterned substrates (STEPS): a new versatile nanofabrication method. Nano Lett. 2012;12(2):527-33. doi:10.1021/nl200426g.

14. Unadkat HV, Hulsman M, Cornelissen K, Papenburg BJ, Truckenmuller RK, Carpenter AE, et al. An algorithm-based topographical biomaterials library to instruct cell fate. Proc Natl Acad Sci U S A. 2011;108(40):16565-70. doi:10.1073/pnas. 11098611081109861108.

15. Gobaa S, Hoehnel S, Roccio M, Negro A, Kobel S, Lutolf MP. Artificial niche microarrays for probing single stem cell fate in high throughput. Nat Methods. 2011;8(11):949-55. doi:10.1038/nmeth. 1732 .

16. Roccio M, Gobaa S, Lutolf MP. High-throughput clonal analysis of neural stem cells in microarrayed artificial niches. Integr Biol. 2012;4(4):391-400. doi:10.1039/c2ib00070a.

17. Davis KA, Burke KA, Mather PT, Henderson JH. Dynamic cell behavior on shape memory polymer substrates. Biomaterials. 2011;32(9):2285-93. doi:10.1016/j.biomaterials.2010.12. 006S0142-9612(10)01543-7.

18. Le DM, Kulangara K, Adler AF, Leong KW, Ashby VS. Dynamic topographical control of mesenchymal stem cells by culture on responsive poly(epsilon-caprolactone) surfaces. Adv Mater. 2011;23(29):3278-83. doi:10.1002/adma.201100821.

19. Gao L, McBeath R, Chen CS. Stem cell shape regulates a chondrogenic versus myogenic fate through Rac1 and N-cadherin. Stem Cells. 2010;28(3):564-72. doi:10.1002/stem.308.

20. Jiang X, Bruzewicz DA, Wong AP, Piel M, Whitesides GM. Directing cell migration with asymmetric micropatterns. Proc Natl Acad Sci U S A. 2005;102(4):975-8. doi:10.1073/pnas. 0408954102.

21. Pholpabu P, Kustra S, Wu H, Balasubramanian A, Bettinger CJ. Lithography-free fabrication of reconfigurable substrate topography for contact guidance. Biomaterials. 2015;39:164-72. doi:10. 1016/j.biomaterials.2014.10.078S0142-9612(14)01142-9.

22. Kiang JD, Wen JH, del Alamo JC, Engler AJ. Dynamic and reversible surface topography influences cell morphology. J Biomed Mater Res A. 2013;101(8):2313-21. doi:10.1002/jbm.a.34543.

23. Kirschner CM, Anseth KS. In situ control of cell substrate microtopographies using photolabile hydrogels. Small. 2013;9(4): 578-84. doi:10.1002/smll.201201841.

24. Chowdhury F, Na S, Li D, Poh YC, Tanaka TS, Wang F, et al. Material properties of the cell dictate stress-induced spreading and differentiation in embryonic stem cells. Nat Mater. 2010;9(1):82-8. doi:10.1038/nmat2563nmat2563.

25. Discher DE, Mooney DJ, Zandstra PW. Growth factors, matrices, and forces combine and control stem cells. Science. 2009;324(5935): 1673-7. doi:10.1126/science.1171643324/5935/1673.

26. Young JL, Engler AJ. Hydrogels with time-dependent material properties enhance cardiomyocyte differentiation in vitro.
Biomaterials. 2011;32(4):1002-9. doi:10.1016/j.biomaterials. 2010.10.020S0142-9612(10)01309-8.

27. Yambe M, Tomiyama H, Hirayama Y, Gulniza Z, Takata Y, Koji Y, et al. Arterial stiffening as a possible risk factor for both atherosclerosis and diastolic heart failure. Hypertens Res. 2004;27(9):625-31.

28. Kloxin AM, Kasko AM, Salinas CN, Anseth KS. Photodegradable hydrogels for dynamic tuning of physical and chemical properties. Science. 2009;324(5923):59-63. doi:10.1126/science. $1169494324 / 5923 / 59$

29. Tibbitt MW, Kloxin AM, Dyamenahalli KU, Anseth KS. Controlled two-photon photodegradation of PEG hydrogels to study and manipulate subcellular interactions on soft materials. Soft Matter. 2010;6(20):5100-8. doi:10.1039/C0SM00174K.

30. Frey MT, Wang YL. A photo-modulatable material for probing cellular responses to substrate rigidity. Soft Matter. 2009;5:1918 24. doi:10.1039/b818104g.

31. Kloxin AM, Benton JA, Anseth KS. In situ elasticity modulation with dynamic substrates to direct cell phenotype. Biomaterials. 2010;31(1):1-8. doi:10.1016/j.biomaterials.2009.09.025S01429612(09)00955-7.

32. Jiang FX, Yurke B, Schloss RS, Firestein BL, Langrana NA. The relationship between fibroblast growth and the dynamic stiffnesses of a DNA crosslinked hydrogel. Biomaterials. 2010;31(6):1199 212. doi:10.1016/j.biomaterials.2009.10.050S0142-9612(09) 01171-5.

33. Tong X, Yang F. Engineering interpenetrating network hydrogels as biomimetic cell niche with independently tunable biochemical and mechanical properties. Biomaterials. 2014;35(6):1807-15. doi:10. 1016/j.biomaterials.2013.11.064S0142-9612(13)01430-0.

34. Winer JP, Oake S, Janmey PA. Non-linear elasticity of extracellular matrices enables contractile cells to communicate local position and orientation. PLoS One. 2009;4(7), e6382. doi:10.1371/journal. pone. 0006382 .

35. Aulisa L, Dong H, Hartgerink JD. Self-assembly of multidomain peptides: sequence variation allows control over cross-linking and viscoelasticity. Biomacromolecules. 2009;10(9):2694-8. doi:10. 1021/bm900634x.

36. Haines-Butterick L, Rajagopal K, Branco M, Salick D, Rughani R, Pilarz $\mathrm{M}$, et al. Controlling hydrogelation kinetics by peptide design for three-dimensional encapsulation and injectable delivery of cells. Proc Natl Acad Sci U S A. 2007;104(19):7791-6. doi:10.1073/ pnas.0701980104.

37. Zhang S, Greenfield MA, Mata A, Palmer LC, Bitton R, Mantei JR, et al. A self-assembly pathway to aligned monodomain gels. Nat Mater. 2010;9(7):594-601. doi:10.1038/nmat2778nmat2778.

38. Olsen BD, Kornfield JA, Tirrell DA. Yielding behavior in injectable hydrogels from telechelic proteins. Macromolecules. 2010;43(21): 9094-9. doi:10.1021/ma101434a.

39. Stephanopoulos N, Ortony JH, Stupp SI. Self-assembly for the synthesis of functional biomaterials. Acta Mater. 2013;61(3):91230. doi:10.1016/j.actamat.2012.10.046.

40. Gaffey AC, Chen MH, Venkataraman CM, Trubelja A, Rodell CB, Dinh PV, et al. Injectable shear-thinning hydrogels used to deliver endothelial progenitor cells, enhance cell engraftment, and improve ischemic myocardium. J Thorac Cardiovasc Surg. 2015;150(5): 1268-77. doi:10.1016/j.jtcvs.2015.07.035S0022-5223(15)01252-0.

41. Jeon $\mathrm{O}$, Alsberg E. Photofunctionalization of alginate hydrogels to promote adhesion and proliferation of human mesenchymal stem cells. Tissue Eng A. 2013;19(11-12):1424-32. doi:10.1089/ten. TEA.2012.0581.

42. Robertus J, Browne WR, Feringa BL. Dynamic control over cell adhesive properties using molecular-based surface engineering strategies. Chem Soc Rev. 2010;39(1):354-78. doi:10.1039/ b906608j.

43. Holland TA, Tabata Y, Mikos AG. Dual growth factor delivery from degradable oligo(poly(ethylene glycol) fumarate) hydrogel 
scaffolds for cartilage tissue engineering. J Control Release. 2005;101(1-3):111-25. doi:10.1016/j.jconrel.2004.07.004.

44. Yonet-Tanyeri N, Rich MH, Lee M, Lai MH, Jeong JH, DeVolder RJ, et al. The spatiotemporal control of erosion and molecular release from micropatterned poly(ethylene glycol)-based hydrogel. Biomaterials. 2013;34(33):8416-23. doi:10.1016/j.biomaterials. 2013.07.026S0142-9612(13)00808-9.

45. Bajpai AK, Shukla SK, Bhanu S, Kankane S. Responsive polymers in controlled drug delivery. Prog Polym Sci. 2008;33(11):1088118. doi:10.1016/j.progpolymsci.2008.07.005.

46. Qiu Y, Park K. Environment-sensitive hydrogels for drug delivery. Adv Drug Deliv Rev. 2001;53(3):321-39.

47. Ali OA, Doherty E, Mooney DJ, Emerich D. Relationship of vaccine efficacy to the kinetics of DC and T-cell responses induced by PLG-based cancer vaccines. Biomatter. 2011;1(1):66-75. doi:10. 4161/biom.1.1.1627716277.

48. Brack AS, Conboy IM, Conboy MJ, Shen J, Rando TA. A temporal switch from notch to Wnt signaling in muscle stem cells is necessary for normal adult myogenesis. Cell Stem Cell. 2008;2(1):50-9. doi:10.1016/j.stem.2007.10.006S1934-5909(07)00223-8.

49. Hori Y, Stern PJ, Hynes RO, Irvine DJ. Engulfing tumors with synthetic extracellular matrices for cancer immunotherapy. Biomaterials. 2009;30(35):6757-67. doi:10.1016/j.biomaterials. 2009.08.037S0142-9612(09)00899-0.

50. Olivera-Martinez I, Schurch N, Li RA, Song J, Halley PA, Das RM, et al. Major transcriptome re-organisation and abrupt changes in signalling, cell cycle and chromatin regulation at neural differentiation in vivo. Development. 2014;141(16):3266-76. doi:10.1242/ dev.112623dev.112623

51. Ali OA, Huebsch N, Cao L, Dranoff G, Mooney DJ. Infectionmimicking materials to program dendritic cells in situ. Nat Mater. 2009;8(2):151-8. doi:10.1038/nmat2357nmat2357.

52. DeForest CA, Tirrell DA. A photoreversible protein-patterning approach for guiding stem cell fate in three-dimensional gels. Nat Mater. 2015;14(5):523-31. doi:10.1038/nmat4219nmat4219.

53. Wong JY, Leach JB, Brown XQ. Balance of chemistry, topography, and mechanics at the cell-biomaterial interface: Issues and challenges for assessing the role of substrate mechanics on cell response. Surf Sci. 2004;570(1-2):119-33. doi:10.1016/j.susc.2004. 06.186.

54. Algahtani MS, Scurr DJ, Hook AL, Anderson DG, Langer RS, Burley JC, et al. High throughput screening for biomaterials discovery. J Control Release. 2014;190:115-26. doi:10.1016/j.jconrel. 2014.06.045.

55. Davies MC, Alexander MR, Hook AL, Yang J, Mei Y, Taylor M, et al. High throughput surface characterization: a review of a new tool for screening prospective biomedical material arrays. J Drug Target. 2010;18(10):741-51. doi:10.3109/1061186X.2010.521941.

56. Hook AL, Anderson DG, Langer R, Williams P, Davies MC, Alexander MR. High throughput methods applied in biomaterial development and discovery. Biomaterials. 2010;31(2):187-98. doi: 10.1016/j.biomaterials.2009.09.037.

57. Mei Y, Hollister-Lock J, Bogatyrev SR, Cho SW, Weir GC, Langer $\mathrm{R}$, et al. A high throughput micro-array system of polymer surfaces for the manipulation of primary pancreatic islet cells. Biomaterials. 2010;31(34):8989-95. doi:10.1016/j.biomaterials.2010.08.029.

58. Pernagallo S, Unciti-Broceta A, Diaz-Mochon JJ, Bradley M. Deciphering cellular morphology and biocompatibility using polymer microarrays. Biomed Mater. 2008;3(3):034112. doi:10.1088/ 1748-6041/3/3/034112.

59. Yang J, Mei Y, Hook AL, Taylor M, Urquhart AJ, Bogatyrev SR, et al. Polymer surface functionalities that control human embryoid body cell adhesion revealed by high throughput surface characterization of combinatorial material microarrays. Biomaterials. 2010;31(34):8827-38. doi:10.1016/j.biomaterials.2010.08.028.
60. Hook AL, Chang CY, Yang J, Scurr DJ, Langer R, Anderson DG, et al. Polymer microarrays for high throughput discovery of biomaterials. J Vis Exp. 2012;59, e3636. doi:10.3791/3636.

61. Lamers E, van Horssen R, te Riet J, van Delft FC, Luttge R, Walboomers XF, et al. The influence of nanoscale topographical cues on initial osteoblast morphology and migration. Eur Cell Mater. 2010;20:329-43.

62. Hulsman M, Hulshof F, Unadkat H, Papenburg BJ, Stamatialis DF, Truckenmuller R, et al. Analysis of high-throughput screening reveals the effect of surface topographies on cellular morphology. Acta Biomater. 2015;15:29-38. doi:10.1016/j.actbio.2014.12. 019S1742-7061(14)00583-2.

63. Nakajima M, Ishimuro T, Kato K, Ko IK, Hirata I, Arima Y, et al. Combinatorial protein display for the cell-based screening of biomaterials that direct neural stem cell differentiation. Biomaterials. 2007;28(6):1048-60. doi:10.1016/j.biomaterials.2006.10.004.

64. Floren M, Tan W. Three-dimensional, soft neotissue arrays as high throughput platforms for the interrogation of engineered tissue environments. Biomaterials. 2015;59:39-52. doi:10.1016/j. biomaterials.2015.04.036S0142-9612(15)00411-1.

65. Memic A, Khademhosseini A. Finding the winning combination. Combinatorial screening of three dimensional niches to guide stem cell osteogenesis. Organogenesis. 2014;10(3):299-302. doi:10. 4161/org.29646.

66. Rape AD, Zibinsky M, Murthy N, Kumar S. A synthetic hydrogel for the high-throughput study of cell-ECM interactions. Nat Commun. 2015;6:8129. doi:10.1038/ncomms9129ncomms9129.

67. Garcia-Cordero JL, Nembrini C, Stano A, Hubbell JA, Maerkl SJ. A high-throughput nanoimmunoassay chip applied to large-scale vaccine adjuvant screening. Integr Biol. 2013;5(4):650-8. doi:10. 1039/c3ib20263a.

68. Liu E, Gordonov S, Treiser MD, Moghe PV. Parsing the early cytoskeletal and nuclear organizational cues that demarcate stem cell lineages. Cell Cycle. 2010;9(11):2108-17.

69. Liu E, Treiser MD, Patel H, Sung HJ, Roskov KE, Kohn J, et al. High-content profiling of cell responsiveness to graded substrates based on combinyatorially variant polymers. Comb Chem High Throughput Screen. 2009;12(7):646-55.

70. Treiser MD, Liu E, Dubin RA, Sung HJ, Kohn J, Moghe PV. Profiling cell-biomaterial interactions via cell-based fluororeporter imaging. Biotechniques. 2007;43(3):361-6. 8 .

71. Treiser MD, Yang EH, Gordonov S, Cohen DM, Androulakis IP, Kohn J, et al. Cytoskeleton-based forecasting of stem cell lineage fates. Proc Natl Acad Sci U S A. 2010;107(2):610-5. doi:10.1073/ pnas.0909597107.

72. Vega SL, Dhaliwal A, Arvind V, Patel PJ, Beijer NR, de Boer J, et al. Organizational metrics of interchromatin speckle factor domains: integrative classifier for stem cell adhesion \& lineage signaling. Integr Biol. 2015;7(4):435-46. doi:10.1039/c4ib00281d.

73. Huang TY, Wu CH, Wang MH, Chen BS, Chiou LL, Lee HS. Cooperative regulation of substrate stiffness and extracellular matrix proteins in skin wound healing of axolotls. Biomed Res Int. 2015;2015:712546. doi:10.1155/2015/712546.

74. Martin HL, Adams M, Higgins J, Bond J, Morrison EE, Bell SM, et al. High-content, high-throughput screening for the identification of cytotoxic compounds based on cell morphology and cell proliferation markers. PLoS One. 2014;9(2), e88338. doi:10.1371/journal. pone.0088338PONE-D-13-36866.

75. Massey AJ. Multiparametric cell cycle analysis using the operetta high-content imager and harmony software with PhenoLOGIC. PLoS One. 2015;10(7), e0134306. doi:10.1371/journal.pone. 0134306PONE-D-15-17844.

76. Peppard JV, Rugg C, Smicker M, Dureuil C, Ronan B, Flamand O, et al. Identifying small molecules which inhibit autophagy: a phenotypic screen using image-based high-content cell analysis. Curr 
Chem Genom Transl Med. 2014;8 Suppl 1:3-15. doi:10.2174/ 2213988501408010003CCGTM-8-3.

77. Solmesky LJ, Shuman M, Goldsmith M, Weil M, Peer D. Assessing cellular toxicities in fibroblasts upon exposure to lipid-based nanoparticles: a high content analysis approach. Nanotechnology. 2011;22(49):494016. doi:10.1088/0957-4484/22/49/494016.

78. Kilner J, Waby JS, Chowdry J, Khan AQ, Noirel J, Wright PC, et al. A proteomic analysis of differential cellular responses to the shortchain fatty acids butyrate, valerate and propionate in colon epithelial cancer cells. Mol BioSyst. 2012;8(4):1146-56. doi:10.1039/ c1mb05219e.

79. Radio NM, Breier JM, Shafer TJ, Mundy WR. Assessment of chemical effects on neurite outgrowth in PC12 cells using high content screening. Toxicol Sci. 2008;105(1):106-18. doi:10.1093/ toxsci/kfn 114 .

80. Sirenko O, Crittenden C, Callamaras N, Hesley J, Chen YW, Funes $\mathrm{C}$, et al. Multiparameter in vitro assessment of compound effects on cardiomyocyte physiology using iPSC cells. J Biomol Screen. 2013;18(1):39-53. doi:10.1177/1087057112457590.

81. Degot S, Auzan M, Chapuis V, Beghin A, Chadeyras A, Nelep C, et al. Improved visualization and quantitative analysis of drug effects using micropatterned cells. J Vis Exp. 2010;46. doi:10.3791/2514.

82. Stockwell SR, Mittnacht S. Workflow for high-content, individual cell quantification of fluorescent markers from universal microscope data, supported by open source software. J Vis Exp. 2014;94. doi:10.3791/51882.

83. Unadkat HV, Groen N, Doorn J, Fischer B, Barradas AM, Hulsman $\mathrm{M}$, et al. High content imaging in the screening of biomaterial-induced MSC behavior. Biomaterials. 2013;34(5): 1498-505. doi:10.1016/j.biomaterials.2012.10.035.

84. Narayan PJ, Dragunow M. High content analysis of histone acetylation in human cells and tissues. J Neurosci Methods. 2010;193(1): 54-61. doi:10.1016/j.jneumeth.2010.08.032.

85. Ren Y, Lin S, Jing Y, Dechow PC, Feng JQ. A novel way to statistically analyze morphologic changes in Dmp1-null osteocytes. Connect Tissue Res. 2014;55 Suppl 1:129-33. doi:10.3109/ 03008207.2014.923879.

86. Ren ZX, Yu HB, Li JS, Shen JL, Du WS. Suitable parameter choice on quantitative morphology of A549 cell in epithelial-mesenchymal transition. Biosci Rep. 2015;35(3). doi:10.1042/BSR20150070.

87. Song HP, Li RL, Zhou C, Cai X, Huang HY. Atractylodes macrocephala Koidz stimulates intestinal epithelial cell migration through a polyamine dependent mechanism. J Ethnopharmacol. 2015;159:23-35. doi:10.1016/j.jep.2014.10.059.

88. Atkuri KR, Stevens JC, Neubert H. Mass cytometry: a highly multiplexed single-cell technology for advancing drug development. Drug Metab Dispos. 2015;43(2):227-33. doi:10.1124/dmd. 114.060798

89. Leipold MD, Newell EW, Maecker HT. Multiparameter phenotyping of human PBMCs using mass cytometry. Methods Mol Biol. 2015;1343:81-95. doi:10.1007/978-1-4939-2963-4 7.

90. Mora J, Given Chunyk A, Dysinger M, Purushothama S, Ricks $\mathrm{C}$, Osterlund $\mathrm{K}$, et al. Next generation ligand binding assaysreview of emerging technologies' capabilities to enhance throughput and multiplexing. AAPS J. 2014;16(6):1175-84. doi:10.1208/s12248-014-9660-1. 